\title{
3-D Molecular Depth Profiling of Biological and Polymeric Materials Using Time- of-Flight Secondary Ion Mass Spectrometry
}

\author{
Paula A. Clark ${ }^{1}$, Elke Tallarek, ${ }^{1,2}$ Daniel Breitenstein ${ }^{2}$, Birgit Hagenhoff, ${ }^{2}$ and Nathan Havercroft ${ }^{3}$ \\ 1. TASCON USA, 100 Red Schoolhouse Road, Chestnut Ridge, NY 10977 \\ 2. TASCON GmbH, Mendelstrasse 1748149 Münster, Germany \\ 3. ION-TOF USA, 100 Red Schoolhouse Road, Chestnut Ridge, NY 10977
}

An increasing number of new nano-structured devices such as organic light emitting diodes, displays, sensors, etc., make use of complex organic molecules organized in specific 2-D and 3-D arrays. Therefore, there is an increasing need for analytical techniques which provide molecular information with high lateral and high depth resolution. This paper highlights the current state-of-art in 3-D molecular analysis of biological and polymeric materials using Time-of-Flight Secondary Ion Mass Spectrometry (ToF-SIMS). In SIMS, a sample is introduced into an ultra-high vacuum chamber and bombarded with a primary ion beam. The impact of the primary ion results in the desorption (sputtering) of neutral species, electrons, and secondary ions from the surface of the sample. The secondary ions are mass analyzed. The advantages of SIMS include high sensitivity, the ability to obtain molecular information, isotopic analysis, imaging, and the analysis of low-atomic number elements such as $\mathrm{H}$ and $\mathrm{Li}[1]$. An exciting advancement in the last $5-10$ years has been the development of cluster primary ion sources $\left(\mathrm{Au}_{\mathrm{n}}{ }^{+}, \mathrm{Bi}_{\mathrm{n}}{ }^{+}, \mathrm{SF}_{5}{ }^{+}, \mathrm{C}_{60}{ }^{+}\right.$, or $\left.\mathrm{Ar}_{\mathrm{n}}{ }^{+}\right)[2,3]$. Relative to atomic primary ion sources, cluster ion sources provide higher secondary ion yields and therefore higher sensitivity. Moreover, $\mathrm{C}_{60}{ }^{+}$, and $\mathrm{Ar}_{\mathrm{n}}{ }^{+}$primary sources cause significantly less chemical damage to the sample and have thus facilitated molecular depth profiling; i.e., the ability to monitor molecular species as a function of depth into the sample. The principle of 3-D ToF-SIMS Molecular Depth Profiling in Dual Beam Mode is presented in Figure 1.

The ToF-SIMS data were obtained on an ION-TOF IV instrument using $\mathrm{Bi}_{3}{ }^{+}$as the analysis beam and $\mathrm{C}_{60}{ }^{+}$as the sputter source (Figure 1) [4].

The detection of both pharmaceutically active and potentially harmful substances in biological cells is important in the medical industry. Historically, investigations on the uptake, the working mechanism, and the metabolism of these compounds depended on labeling the relevant compounds - e.g. by fluorescent dyes or (radioactive) isotopes. ToF-SIMS has the potential to identify species without the need for labeling; however, since ToF-SIMS is a vacuum technique, fixation procedures are used to preserve the intact cellular structures [4]. Figure 2 shows the ToF-SIMS images acquired from rat kidney epithelial cells which were fixed using glutaraldehyde, a fixation protocol that preserves the internal cell structure. Figure 2 shows the epithelial cell surface is dominated by phospholipids $(a-c)$. 
After sputtering with $\mathrm{C}_{60}{ }^{+}(\mathrm{d}-\mathrm{f})$ intense phospholipid signal is found to surround a central area, rich in amino acids. This distribution is attributed to the membrane-rich non-nucleus area of the cells and the protein rich nucleus. In conclusion, the development of $\mathrm{C}_{60}{ }^{+}$and $\mathrm{Ar}_{\mathrm{n}}{ }^{+}$sputter sources has significantly improved 3-D molecular analysis; however, real-world problems must be investigated on a case-by-case basis.

Figure 1. Combining a small spot size analysis

$$
\mathrm{Bi}_{n} \text { Analysis } \quad \mathrm{C}_{60}{ }^{+} \text {or } \mathrm{Ar}_{n}
$$

source (e.g., $\mathrm{Bi}_{\mathrm{n}}{ }^{+}$) with the larger sputter source (e.g., $\mathrm{C}_{60}{ }^{+}$or $\mathrm{Ar}_{\mathrm{n}}{ }^{+}$) in a dual-beam profiling experiment enables 3-D molecular depth profiling with a depth resolution of $<5 \mathrm{~nm}$ and lateral resolution of the order $150 \mathrm{~nm}$ to several microns.

Figure 2. Mass-resolved secondary ion images of the epithelial cells before sputtering $(\mathrm{a}-\mathrm{c})$, within the cell after sputtering with $\mathrm{C}_{60}{ }^{+}(\mathrm{d}-\mathrm{f})$, and $\mathrm{xz}$ sections through the $3-\mathrm{D}$ data along the white line $\mathrm{g}-\mathrm{i})$.

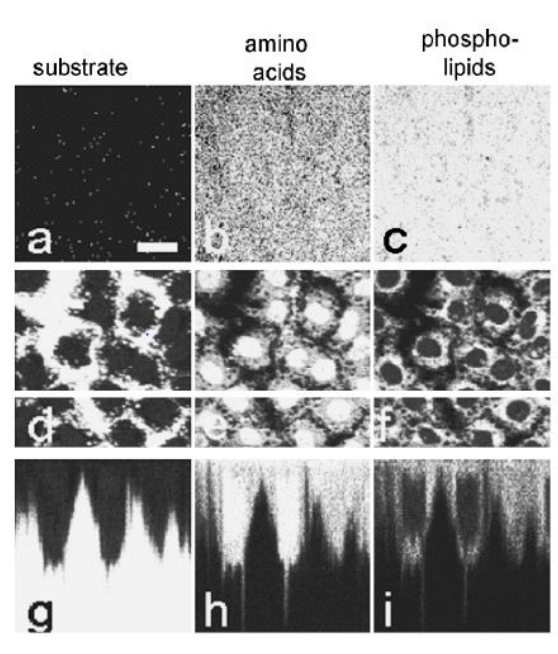

\section{References:}

[1] J. C. Vickerman, Molecular Surface Mass Spectrometry by SIMS, in Surface Analysis - The Principle Techniques, eds. J. A. Vickerman and I. S. Gilmore, (John Wiley and Sons, Ltd., West Sussex) Chapter 4.

[2] S. Rabbani, A. M. Barber, J. S. Fletcher, N. P. Lockyer, J. C. Vickerman, Anal. Chem., 83 (2011), p. 3793.

[3] C. M Mahoney, Mass Spectrometry Reviews, Wiley Periodicals, Inc. (2009).

[4] D. Breitenstein, C. E. Rommel, J. Stolwijk, J. Wegener, and B. Hagenhoff, App. Sur. Sci., 255 (2008), p. 1249. 